pomegranate juice markedly slows the progression of prostate cancer. Their results suggest that pomegranate juice has cytostatic, rather than cytocidal, anticancer activity.

This single-center, open-label phase II clinical trial evaluated 46 men with biochemical recurrence of prostate cancer after surgery and/or radiotherapy. The men drank $8 \mathrm{oz}$ of pomegranate juice daily (containing $570 \mathrm{mg}$ polyphenol gallic acid), and were followed at 3-month intervals until disease progression. Pomegranate juice caused a statistically significant prolongation of mean PSA doubling time, from 15 months to 54 months. In vitro assays also showed that pomegranate juice had antiproliferative and proapoptotic effects on prostate cancer cells, although the authors recognize that the clinical relevance of these results is unclear. They speculate that the beneficial effects of pomegranate juice are mediated by its antioxidant and anti-inflammatory properties.

A prospective, placebo-controlled, randomized trial to determine the effects of two different doses of pomegranate juice on PSA kinetics is underway. The authors also plan to evaluate the performance of serum lipid peroxidation and plasma nitric oxide as biomarkers of oxidative stress, and to identify factors that might mediate the anticancer effects of pomegranate juice.

Original article Pantuck AJ et al. (2006) Phase II study of pomegranate juice for men with rising prostate-specific antigen following surgery or radiation for prostate cancer. Clin Cancer Res 12: 4018-4026

\section{New immunotherapy agent prolongs survival of men with advanced prostate cancer}

Men with advanced prostate cancer have a very limited life expectancy, and the few treatment options available are often accompanied by severe adverse effects. A new class of immunotherapeutic agents based on modified autologous antigen-presenting cells is being developed; these stimulate the patient's own cellular immunity and have minimal toxicity. Promising results have been reported from a phase III trial of one such agent, sipuleucel-T, which targets prostatic acid phosphatase, a prostate-cancer-specific antigen.

In a double-blind, placebo-controlled, randomized trial, 127 men with asymptomatic, metastatic, hormone-refractory prostate cancer received three infusions of either sipuleucel-T or placebo over 4 weeks. Analysis showed that the median survival of men who received sipuleucel-T was 4.5 months longer than that of men who received placebo (25.9 months versus 21.4 months, $P=0.01$ ). The 3 -year survival rate was $34 \%$ in men who received sipuleucel-T, and $11 \%$ in men who received placebo $(P=0.005)$. In addition, men who received sipuleucel-T showed eightfold greater T-cell stimulation (compared with pretreatment levels) than men who received placebo $(P<0.001)$.

Despite these promising results, the trial just failed to meet its primary endpoint of prolonged time to disease progression. The researchers speculate that immunotherapeutic agents such as sipuleucel-T might achieve their biologic effects gradually, and might be more effective in patients with less-aggressive disease.

Original article Small EJ et al. (2006) Placebo-controlled phase III trial of immunologic therapy with sipuleucel-T (APC8015) in patients with metastatic, asymptomatic hormone refractory prostate cancer. J Clin Oncol 24: 3089-3094

\section{Condom catheters are recommended for men without dementia}

Catheter-related urinary tract infection is very common in hospitalized patients and those in long-term care. Although studies have shown that men prefer external, 'condom' catheters to indwelling urethral catheters, there are no data that compare the rate of adverse events associated with each catheter type. Saint et al., therefore, have conducted the first randomized trial that compares external and indwelling catheters in hospitalized men.

Over the 4-year recruitment period, only 75 patients were both eligible and gave consent: 34 were allocated to the condom-catheter group and 41 to the indwelling-catheter group. The mean length of hospital stay was 3 days. Saint et al. found that, after controlling for other risk factors (mainly dementia), the incidence of bacteriuria, symptomatic urinary tract infection, or death was significantly lower in the condomcatheter group than in the indwelling-catheter group $(P=0.04)$. Men without dementia were almost five times more likely to experience these adverse events if they used an indwelling catheter $(P=0.01)$. In addition, significantly 